\title{
Saliency-based 3D convolutional neural network for categorising common focal liver lesions on multisequence MRI
}

Shu-Hui Wang ${ }^{1,2}$, Xin-Jun Han ${ }^{1}$, Jing Du' ${ }^{1}$, Zhen-Chang Wang ${ }^{1}$, Chunwang Yuan ${ }^{3}$, Yinan Chen ${ }^{4,5}$, Yajing Zhu ${ }^{4}$, Xin Dou ${ }^{6}$, Xiao-Wei $\mathrm{Xu}^{4}$, Hui $\mathrm{Xu}^{1^{*}}$ and Zheng-Han Yang ${ }^{1 *}$ (D)

\begin{abstract}
Background: The imaging features of focal liver lesions (FLLs) are diverse and complex. Diagnosing FLLs with imaging alone remains challenging. We developed and validated an interpretable deep learning model for the classification of seven categories of FLLS on multisequence MRI and compared the differential diagnosis between the proposed model and radiologists.

Methods: In all, 557 lesions examined by multisequence MRI were utilised in this retrospective study and divided into training-validation $(n=444)$ and test $(n=113)$ datasets. The area under the receiver operating characteristic curve (AUC) was calculated to evaluate the performance of the model. The accuracy and confusion matrix of the model and individual radiologists were compared. Saliency maps were generated to highlight the activation region based on the model perspective.

Results: The AUC of the two- and seven-way classifications of the model were 0.969 (95\% Cl 0.944-0.994) and from 0.919 (95\% Cl 0.857-0.980) to 0.999 (95\% Cl 0.996-1.000), respectively. The model accuracy (79.6\%) of the sevenway classification was higher than that of the radiology residents $(66.4 \%, p=0.035)$ and general radiologists $(73.5 \%$, $p=0.346$ ) but lower than that of the academic radiologists $(85.4 \%, p=0.291)$. Confusion matrices showed the sources of diagnostic errors for the model and individual radiologists for each disease. Saliency maps detected the activation regions associated with each predicted class.
\end{abstract}

Conclusion: This interpretable deep learning model showed high diagnostic performance in the differentiation of FLLs on multisequence MRI. The analysis principle contributing to the predictions can be explained via saliency maps.

Keywords: Deep learning, MRI, Classification, Focal liver lesion, Model interpretation

\section{Key points}

- AI has the potential to relieve physicians by automating the process.

- This model could accurately classify common liver masses on multisequence MRI.

\footnotetext{
*Correspondence: mr_xuhui@163.com; yangzhenghan@vip.163.com ${ }^{1}$ Department of Radiology, Beijing Friendship Hospital, Capital Medical University, No. 95 Yongan Road, Xicheng District, Beijing 100050, People's Republic of China

Full list of author information is available at the end of the article
}

- Different MRI scanners and liver background did not affect the model performance.

- Saliency maps could explain model decision-making and let radiologists verify the diagnosis.

\section{Introduction}

With the development of imaging technology, most focal liver lesions (FLLs) can be detected accurately by MRI [1]. Nevertheless, diagnosing FLLs with imaging alone remains a challenge. The imaging features of FLLs are 
diverse and complex, and different lesion features overlap. Atypical characteristics in some common lesions make the diagnosis challenging, including atypical morphologic features, atypical location or lesions that may mimic other primary liver tumours [2]. Maximising the imaging diagnosis accuracy of FLLs is paramount in avoiding unnecessary biopsies [3] and optimal patient management. Meanwhile, the evaluation and analysis of medical images are generally subjective and may possibly be affected by the experience of radiologists with various levels of specialisation [4]. Artificial intelligence (AI) could aid doctors in diagnosing FLLs and potentially be useful in both helping inexperienced physicians and bridging the gap between novice and expert radiologists [5].

As a strategy for AI, convolutional neural network (CNN)-based deep learning systems have been widely considered in radiology $[6,7]$. Different from radiologists who diagnose diseases through radiological features and conventional machine learning algorithms that rely on handcrafted features, $\mathrm{CNNs}$ can automatically learn complex features from medical images [8]. Therefore, by learning from a sufficiently large amount of training data, CNNs may accurately categorise FLLs without relying on the experience of radiologists.

There have been several AI studies on FLL classification, but they have mainly focused on two-dimensional (2D) CNN models $[9,10]$ or have been based on computed tomography (CT) images [11]. There is a lack of research on three-dimensional (3D) $\mathrm{CNNs}$ based on MRI data. Compared with 2D CNNs, 3D CNNs based on magnetic resonance imaging (MRI) data can resample tumour slices more effectively, improve the sample size, obtain rich 3D tumour information and capture tissue characteristics more accurately $[12,13]$. In addition, previous studies still lack interpretability for the "black box". At present, the output of a 3D CNN heat map is still technically difficult to interpret, which makes it impossible to verify whether the model follows some aspects of human expert knowledge. The aim of our study was to potentially relieve physicians and staff of the need to carry out primary diagnosis by automating the process and thus lighten the burden on radiologists. We developed an interpretable 3D CNN based on multiple sequences for the classification of seven categories of common FLLs and validated its performance compared to radiologists with various levels of specialisation.

\section{Material and methods}

This retrospective study was approved by the institutional Ethical Review Committee (Approval Number: 2019P2-230-01) and the requirement for written informed consent was waived. In this study, a sample size was not prespecified. Nonemployee or nonconsultant authors analysed and controlled the data.

\section{Patients and diseases}

There were 445 study patients, each with one anonymised liver MRI (study) acquired between January 2017 and December 2019. Studies were obtained from our institution's picture archiving and communication system (PACS) according to the procedures detailed in Additional file 1: Fig. S1.

The inclusion criteria were as follows: (1) participants underwent unenhanced and enhanced liver MRI inspection; (2) participants had one of the following common FLLs, including liver cyst, cavernous haemangioma (HEM), hepatic abscess (HEP), focal nodular hyperplasia $(\mathrm{FNH})$, hepatocellular carcinoma (HCC), intrahepatic cholangiocarcinoma (ICC) and hepatic metastasis (MET); and (3) up to one imaging study per patient was included, and up to six lesions were used in each study. We chose only one type of lesion from each case for the following evaluations. The exclusion criteria were as follows: (1) patients with MRI studies of insufficient image quality. (2) Participants who had received treatment related to the lesion before MRI inspection; and (3) diffuse lesions for which the boundary could not be delineated or malignancies involving the portal vein, hepatic vein or adjacent organs. Most malignant tumours were confirmed by histopathology, while other malignancies and benign tumours were diagnosed by follow-up reports that were supported by two radiologists (with 10 and 20 years of experience, respectively) for 3-12 months.

\section{MRI acquisition protocol}

Abdominal MRI was performed on the patient in a supine position using 1.5-T and 3-T MRI scanners, including Siemens (Prisma, TrioTim), GE Healthcare (GE 750w, Signa) and Philips (Ingenia) systems. T2-weighted imaging (T2WI), diffusion-weighted imaging (DWI) ( $b$ value: $800 \mathrm{~s} / \mathrm{mm}^{2}$ ) and apparent diffusion coefficient (ADC) mapping from standard institutional liver MRI protocols were performed with acquisition times of 2-2.5 min. All the unenhanced sequences and enhanced phases were acquired according to the institutional liver MRI protocol. Imaging parameters varied across different scanners and time frames. The parameters used to acquire the images are summarised in Table 1 . Contrast-enhanced T1WI sequences were used with acquisition times of $12-18$ s. All patients underwent MRI using gadobenate dimeglumine, which was intravenously injected at a dosage of $0.1 \mathrm{mmol} / \mathrm{kg}$ (maximum dose, $20 \mathrm{~mL}$ ) and a rate of $2 \mathrm{~mL} / \mathrm{s}$ followed by a normal saline flush $(50 \mathrm{~mL}$ at $2 \mathrm{~mL} / \mathrm{s}$ ). Except for precontrast T1WI, T2WI, DWI and ADC mapping, postcontrast images were analysed, 
Table 1 Image acquisition

\begin{tabular}{|c|c|c|c|}
\hline MRI scanners & Sequences & & \\
\hline \multicolumn{4}{|l|}{ 3.0 T MRI } \\
\hline & $\mathrm{TR}, \mathrm{T} 2 \mathrm{WI}$ & $\mathrm{DWI} b=0.800$ & $\begin{array}{l}\text { LAVA/ } \\
\text { VIBE/ } \\
\text { eTHRIVE }\end{array}$ \\
\hline $\mathrm{TR}(\mathrm{ms})$ & $\begin{array}{l}\text { 2-3respratory } \\
\text { cycles }\end{array}$ & 3000 & Minimum \\
\hline TE (ms) & 85 & Minimum & Minimum \\
\hline Flip angle $\left(^{\circ}\right)$ & 150 & 90 & 15 \\
\hline Matrix & $288 \times 224$ & $128 \times 128$ & $\begin{array}{l}288 \times 172- \\
320 \times 216\end{array}$ \\
\hline $\mathrm{FOV}(\mathrm{mm})$ & $380-420$ & $380-420$ & $380-420$ \\
\hline Echo train length & 16 & 128 & - \\
\hline Thickness, mm & $6-8$ & $6-8$ & $3-4$ \\
\hline
\end{tabular}

$T R$ respiratory triggered, $T E$ echo time

including the late arterial phase (LAP, 15-20 s postinjection), portal venous phase (PVP, 60-70 s postinjection) and delayed phase (DP, 3-5 min postinjection).

\section{Model construction and evaluation based on CNN algorithm}

There were two groups of classification tasks. The first group of tasks was to classify all the tumours into two categories: benign and malignant. The second group of tasks divided the lesions into seven categories as follows: 0 , cyst; 1 , FNH; 2, haemangioma; 3 , abscess; 4, HCC; 5 , ICC; and 6, metastasis. Here, 0, 1, 2 and 3 indicate benign lesions, while 4, 5 and 6 indicate malignancies. A multitask framework has been demonstrated to improve learning efficiency, potential prediction accuracy and overfitting problems for task-specific models.

\section{Data pre-processing}

MR images were downloaded from the PACS and stored as Digital Imaging and Communications in Medicine (DICOM) format. The images were then converted to NIFTI format to hide patient information. The liver tumours were manually segmented by two subspecialtytrained radiologists with an average of 9-year experience in abdominal diagnosis. The outline of tumour on all sequences was drawn in our self-developed module in the 3D-Slicer software (version 4.8.1, Harvard University, Boston, MA, USA). It could provide more valuable information of the tumour region. When there was a discrepancy between the two radiologists on whether the region was a lesion or on the lesion type, a joint review was performed until a consensus was reached for a final decision.

\section{Image processing}

Seven-sequence (T2WI, DWI, ADC, T1WI, LAP, PVP, DP) images and the matched annotated images were resampled at the same voxel spacing of $[1,1,1]$ with the nearest neighbour interpolation algorithm. We normalised the intensity of MRI images to the range of [0, 1]. For lesions with different diameters, the cropping strategy was different. Lesions smaller than $16 \mathrm{~mm}$ were directly cropped to $32 * 32 * 32 \mathrm{~mm}$, lesions larger than $16 \mathrm{~mm}$ but smaller than $32 \mathrm{~mm}$ were cropped to $64 * 64$ * $64 \mathrm{~mm}$, and the remaining lesions were cropped by dilating the area of the lesion. (The dilation size was randomly changed during the training phase.) Then, the cropped patch images with the target lesion and the matched annotated images were resised to $64 * 64 * 64(\mathrm{~mm})$ using bilinear interpolation and the nearest neighbour interpolation algorithms. The cropped annotated images were processed using the Gaussian blur method. Online data augmentation was applied, which included rotation, flipping, shifting, scaling, lighting alteration and Gaussian noise addition.

\section{Model training}

Our deep learning model was trained on a GeForce GTX 1080Ti (NVIDIA) graphic processing unit using Python 3.7 and PyTorch 1.4.0. The architecture of the model is illustrated in Fig. 1. We applied a 3D extension of the 2D ResNet-18 as the backbone [14]. For each sequence, the MRI image and the matched Gaussian annotated image were fed into a 3D ResNet-18 network, and then a feature representation was obtained. All seven feature representations were concatenated as one fused feature. The feature representations of T2WI, DWI and ADC images were concatenated as one fused feature, which, together with the fused feature of all seven sequences, was used for binary classification (fused feature 2). The feature representations of LAP, PVP and DP images were concatenated as another fused feature, which, together with the fused feature of all seven sequences, was used for the seven-way classification (fused feature 7). To obtain the classification results of the two tasks, both fused feature 2 and fused feature 7 were input into fully connected (FC) layers. The cross-validation method split the dataset of the development cohort into training and validation datasets, and fivefold cross-validation was used.

\section{Model evaluation}

The performance of the model was evaluated on an unseen test dataset containing 113 lesions. For each of the five experiments, the model showing the best performance on the validation dataset was selected. The five selected models were used to infer the two-way (benign 


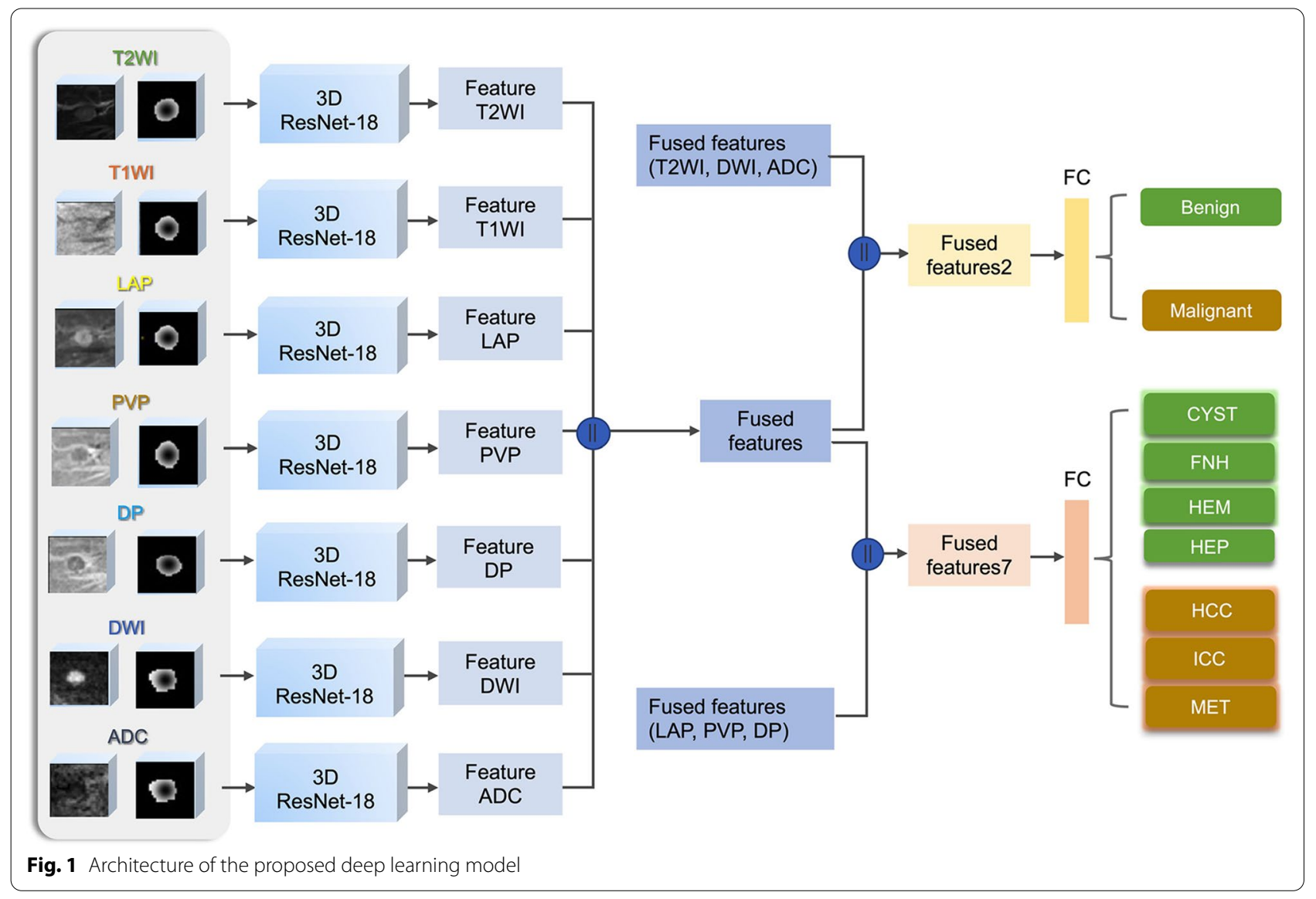

and malignant) and seven-way classification of the test data. The average predicted result of the five experiments on the test data served as the final result. The general demographics of the patients, lesion type, lesion size and MRI scanner were also analysed. We evaluated the influence of liver background (cirrhosis and fatty liver) on the model classification performance. Finally, the model outputs saliency maps to explain the analysis principle.

\section{Comparison to radiologist performance}

Test data were anonymised and independently presented to three categories of radiologists, including two radiology residents (with 1 and 2 years of experience), two general radiologists (with 8 and 12 years of experience) and two academic radiologists (with 17 years and 22 years of experience). All doctors routinely read liver MRI images. ITK-SNAP (version 3.6.0, www.itksnap.org) was used to read images that contained lesions and their surrounding liver parenchyma. Radiologists were given the same MRI sequences available to the AI system.

\section{Statistical analysis}

The characteristics of the development and test datasets are described as follows: continuous variables are expressed as the mean $\pm \mathrm{SD}$ or as the median with interquartile range (IQR) according to the normality of the data; categorical variables are expressed as frequencies (percentage, \%). Student's $t$ test or Kruskal-Wallis test was used for continuous variables, and Chi-square test or Fisher exact test was used for categorical variables.

The area under the receiver operating characteristic (ROC) curve (AUC), accuracy, sensitivity, specificity, positive predictive value (PPV), negative predictive value (NPV), positive diagnostic likelihood ratio (PLR) and negative diagnostic likelihood ratio (NLR) were also calculated. Additionally, 95\% confidence intervals (CIs) were calculated with the modified Wilson method. Different liver backgrounds were compared using Pearson's chi-squared test and Fisher's exact test. The accuracy with $95 \%$ CI was used to compare the difference in diagnostic performance between the model and the radiologists. Interreader agreement was assessed using Cohen's kappa statistic. Confusion matrices were plotted to evaluate the strengths and 
weaknesses of the model and the radiologists. $p<0.05$ was regarded as statistically significant.

\section{Results \\ Patient demographics}

A total of 445 participants were divided into development $(n=356)$ and test $(n=89)$ datasets. There was no significant difference in age $(p=0.795)$ or sex $(p=0.647)$ between the development and test datasets, nor was there a significant difference in tumour type, size or MRI scanner $(p>0.05)$ (Table 2).

\section{Deep learning model performance}

The model showed high performance in the test dataset with 113 lesions, with an average AUC of 0.969 in the two-way classification and $0.919(0.857-0.980)$ to 0.999 (0.996-1.000) in the seven-way classification (Fig. 2). The accuracy, sensitivity, specificity, PPV, NPV, PLR and NLR for each lesion category, determined using the test data, are described in Table 3. The model was found to perform well in diagnosing cysts and HCCs, with median accuracies of $0.991(0.951,1.000)$ and $0.991(0.952,1.000)$, but poorly in diagnosing metastases and abscesses, with median accuracies of $0.805(0.723,0.868)$ and 0.885 $(0.813,0.932)$. The median model sensitivity for the seven categories ranged from $0.909(0.623-0.995)$ to 1.000 (0.806-1.000), the specificity ranged from 0.781 (0.689$0.852)$ to 0.990 (0.944-0.999), and the PPV ranged from $0.432(0.287-0.591)$ to $0.941(0.730-0.997)$.

There were no effects of liver background on model performance. In the two-way classification of the model, the accuracy rates in patients with and without liver cirrhosis in the test cohort were $100.0 \%$ and $87.0 \%$, respectively $(p=0.358)$. The accuracy rates in patients with and without fatty liver were $95.7 \%$ and $86.7 \%$, respectively $(p=0.401)$. In the seven-way classification of the model, the accuracy rates in patients with and without liver cirrhosis in the test cohort were $100.0 \%$ and $77.0 \%$, respectively $(p=0.116)$. The accuracy rates in patients with and without fatty liver were $65.2 \%$ and $83.3 \%$, respectively $(p=0.102)$.

\section{Deep learning model performance compared to radiologist performance}

We compared the performance of the model with that of three categories of radiologists. The performance of the model (90 lesions correct of 113 lesions; mean correct percentage across participants, $79.6 \%$ ) was better than that of the radiology residents (76-78 lesions correct of 113 lesions [67-69\%; mean correct percentage across participants, $68 \%])(p=0.035)$ and general radiologists (80-88 lesions correct of 113 lesions [71-78\%; mean correct percentage across participants, $74 \%])(p=0.346)$.
The accuracy of the model was lower than that of the academic radiologists (96-98 lesions correct of 113 lesions [85-87\%; mean percent correct across participants, $86 \%])(p=0.291)$ (Fig. 3). There was a statistically significant difference in diagnostic performance between the model and radiology residents but not between the model and general or academic radiologists. The agreement was then measured by comparing radiologists within the same specialisation level (Table 4). Two radiologists in the same category showed good consistency (kappa $>0.75, p<0.01$ ).

\section{Evaluating radiologist and model errors}

We evaluated the differential diagnosis distribution of the model and radiologists by means of confusion matrices. Confusion matrices between the model and academic radiologists resembled each other, but in some cases, the model and radiologists made different types of errors. Fewer errors occurred with increasing radiologist specialisation. Radiologists with various levels of specialisation may mistake one certain type of lesion for another. All radiologists made errors in some cases of $\mathrm{HCC}$, but the model did not make the same error (Fig. 4). The average number of model errors was 23 . The model performed well in diagnosing HCC without any mistakes. The model performed poorly in diagnosing FNH among benign lesions and metastasis among malignant lesions. Of 17 metastasis cases, 10 were misidentified, 4 of which were identified as abscesses.

\section{Saliency map}

We selected example saliency maps from seven categories of the test set. Red highlights the activation region of the radiologic imaging feature more associated with the predicted class (Fig. 5). For cysts, the model focused on T2 hyperintensity and T1 hypointensity without contrast enhancement. For haemangioma, the model fixes its attention on discontinuous peripheral nodular enhancement, which progresses in a centripetal direction. For $\mathrm{FNH}$, the model focused on intense arterial hyperenhancement, with near isointensity on the PV and slow gradual enhancement of the central scar. For abscesses, the model focused on restricted diffusion and the typical pattern of peripheral enhancement. For HCC, the model directed its attention to strong arterial enhancement, washout on PVP and DP and capsular enhancement on DP. For ICC, the model directed its attention to rim arterial phase hyperenhancement and delayed central enhancement. For MET, the model concentrated on restricted diffusion and rim hyperenhancement.

Figure 6 shows the weight of each sequence/phase in differential diagnosis. Red colour in saliency maps highlights more important sequence/phase. The importance 
Table 2 Data and patient characteristics

\begin{tabular}{|c|c|c|c|c|}
\hline Characteristic & Total & Development dataset & Test dataset & $p$ value \\
\hline No. of patients & 445 & 356 & 89 & \\
\hline Age (year) & 58 & 58 & 57 & 0.795 \\
\hline Median (IQR) & $(48.00,64.00)$ & $(47.00,64.00)$ & $(51.00,63.00)$ & \\
\hline Sex & & & & 0.647 \\
\hline Male & $272(100 \%)$ & $221(84.4 \%)$ & $50(15.6 \%)$ & \\
\hline Age (year) & 58 & 58 & 59 & 0.238 \\
\hline Median (IQR) & $(49.25,64.00)$ & $(47.75,64.00)$ & $(54.00,65.75)$ & \\
\hline Female & $183(100 \%)$ & $144(78.7 \%)$ & $39(21.3 \%)$ & \\
\hline Age (year) & 56 & 57 & 56 & 0.329 \\
\hline Median (IQR) & $(46.00,63.00)$ & $(47.00,63.00)$ & $(37.50,62.50)$ & \\
\hline Lesion number (\%) & 557 & $444(79.7 \%)$ & $113(20.3 \%)$ & \\
\hline Lesion diameter & 26.8 & 27.3 & 24 & 0.418 \\
\hline Median (IQR) & $(16.80,41.50)$ & $(16.75,41.70)$ & $(17.10,36.38)$ & \\
\hline Lesion type & & & & 0.907 \\
\hline \multicolumn{5}{|l|}{ Cyst } \\
\hline Lesion number (\%) & $86(100 \%)$ & $70(80.7 \%)$ & $16(19.3 \%)$ & \\
\hline Lesion diameter & 12.8 & 12.75 & 12.8 & 0.898 \\
\hline Median (IQR) & $(8.65,22.50)$ & $(8.30,23.43)$ & $(9.80,15.50)$ & \\
\hline \multicolumn{5}{|l|}{ Haemangioma } \\
\hline Lesion number (\%) & $101(100 \%)$ & 79 (78.2\%) & $22(21.8 \%)$ & \\
\hline Lesion diameter & 19.7 & 19.5 & 20.1 & 0.815 \\
\hline Median (IQR) & $(15.30,32.60)$ & $(15.55,32.65)$ & $(15.05,25.02)$ & \\
\hline \multicolumn{5}{|l|}{ FNH } \\
\hline Lesion number (\%) & $57(100 \%)$ & $46(80.7 \%)$ & $11(19.3 \%)$ & \\
\hline Lesion diameter & $34.13 \pm 12.89$ & $35.35 \pm 13.24$ & $28.79 \pm 10.28$ & 0.148 \\
\hline \multicolumn{5}{|l|}{ Mean \pm SE } \\
\hline \multicolumn{5}{|l|}{ Abscess } \\
\hline Lesion number (\%) & $88(100 \%)$ & $67(76.1 \%)$ & $21(23.9 \%)$ & \\
\hline Lesion diameter & $62.98 \pm 31.26$ & $65.20 \pm 32.23$ & $56.31 \pm 29.10$ & 0.479 \\
\hline \multicolumn{5}{|l|}{ Mean \pm SE } \\
\hline \multicolumn{5}{|l|}{ HCC } \\
\hline Lesion number (\%) & $78(100 \%)$ & $63(80.7 \%)$ & $15(19.3 \%)$ & \\
\hline Lesion diameter & 28.9 & 29.35 & 26.8 & 0.537 \\
\hline Median (IQR) & $(23.20,45.90)$ & $(23.45,46.65)$ & $(22.00,38.20)$ & \\
\hline \multicolumn{5}{|l|}{ ICC } \\
\hline Lesion number (\%) & $70(100 \%)$ & $59(84.3 \%)$ & $11(15.7 \%)$ & \\
\hline Lesion diameter & 52.45 & 51.15 & 63.55 & 0.323 \\
\hline Median (IQR) & $(30.72,69.45)$ & $(29.43,66.65)$ & $(47.12,94.50)$ & \\
\hline \multicolumn{5}{|l|}{ Metastasis } \\
\hline Lesion number (\%) & $77(100 \%)$ & $60(77.9 \%)$ & $17(22.1 \%)$ & \\
\hline Lesion diameter & 28.55 & 28.8 & 28.1 & 1 \\
\hline Median (IQR) & $(20.18,38.32)$ & $(19.55,38.75)$ & $(27.80,28.80)$ & \\
\hline Manufacturer and model & & & & 0.499 \\
\hline GE Signa & $126(100 \%)$ & 99 (78.6\%) & $27(21.4 \%)$ & \\
\hline GE Discovery MR750w & $62(100 \%)$ & $46(69.7 \%)$ & $16(30.3 \%)$ & \\
\hline Philips Ingenia & $58(100 \%)$ & $50(86.2 \%)$ & $8(13.8 \%)$ & \\
\hline Siemens prisma & $50(100 \%)$ & $42(84.0 \%)$ & $8(16 \%)$ & \\
\hline Siemens TrioTim & 149 (100\%) & 119 (79.9\%) & $30(20.1 \%)$ & \\
\hline
\end{tabular}

IQR interquartile range 

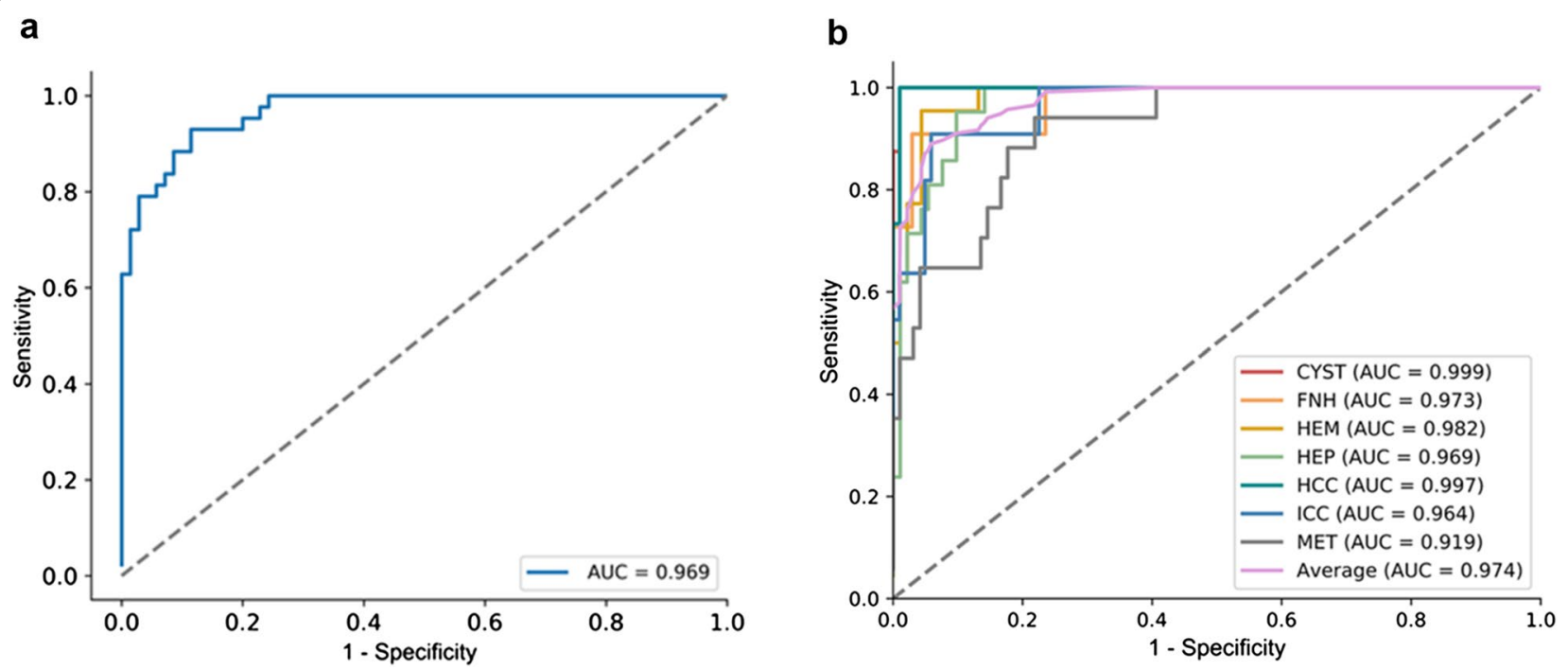

Fig. 2 ROC curves of the (a) two-way and (b) seven-way deep learning model classification

Table 3 Deep learning model diagnostic performance in the two-way and seven-way classification on the test dataset

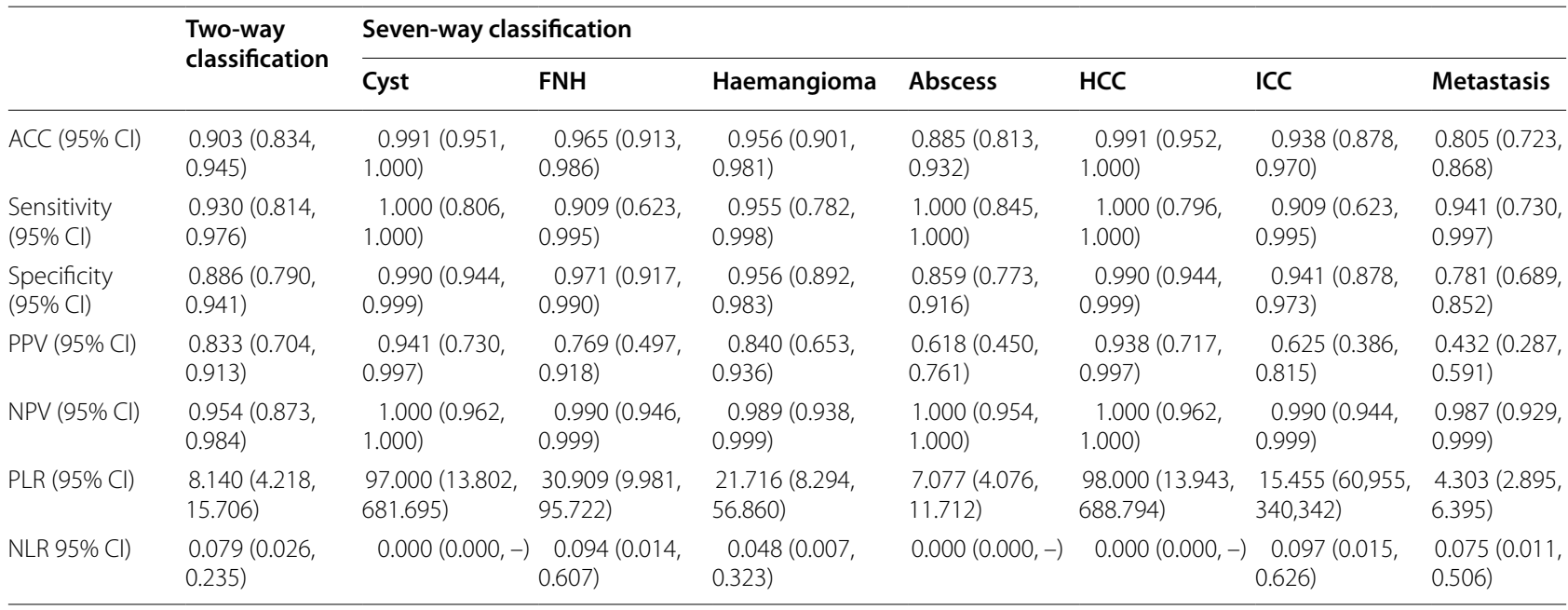

ACC accuracy, PLR positive likelihood ratio, NLR negative likelihood ratio, NPV negative predictive value, PPV positive predictive value

of each sequence/phase in the analysis of seven categories of FLLs is variable.

\section{Discussion}

We developed an interpretable 3D CNN-based classification model for seven categories of common FLLs, using seven sequences and outputted saliency maps to interpret the principle of the model decision-making. The model showed good performance, with an AUC of 0.969 in two-way classification and from $0.919(0.857-0.980)$ to $0.999(0.996-1.000)$ in seven-way classification.
The accuracy of our model was higher than that of the radiologist residents but slightly lower than that of the academic radiologists. The evaluation of medical images by radiologists is subjective and possibly influenced by personal experience. To improve the accuracy and consistency of radiologists and reduce the variability of interpretation, the Liver Imaging Reporting and Data System (LI-RADS) is constantly updated [15-20]. Even so, the diagnostic consistency among radiologists is still variable [21-24]. Kierans et al. [24] demonstrated that by using LI-RADS 2017, the interreader agreement for major features was moderate $(k=0.661)$, 


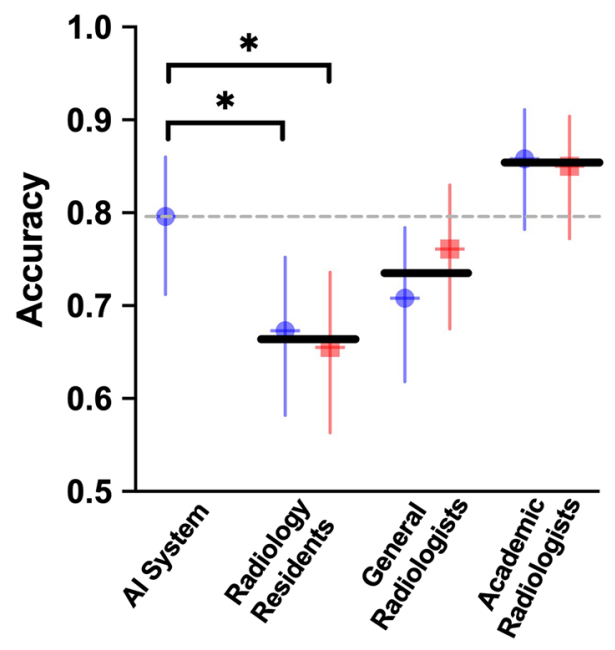

Fig. 3 Performance of the model compared to that of radiologists with various levels of specialisation. Performance is measured as accuracy. Each point represents a single radiologist, and the horizontal line represents the mean across each radiologist category. The horizontal dashed line is the performance of the model. Error bars represent $95 \%$ binomial probability Cls. *There was a significant difference in performance between the model and two types of residents $(p<0.05)$

Table 4 Consistency evaluation between radiologists in the same category

\begin{tabular}{llll}
\hline & Kappa & $\boldsymbol{Z}$ score & $\boldsymbol{p}$ value \\
\hline Radiology residents & 0.915 & 22.49 & $<0.01$ \\
General radiologists & 0.776 & 18.867 & $<0.01$ \\
Academic radiologists & 0.802 & 20.471 & $<0.01$ \\
\hline
\end{tabular}

and ancillary features were poor to fair $(k=0.257$ 0.436) [24]. Our model might maintain diagnostic consistency, help inexperienced physicians, improve the diagnostic accuracy of general radiologists and radiology residents, reduce the workload of academic radiologists and bridge the diagnostic gap between novice and expert radiologists and among different hospitals.

Our model showed good performance in diagnosing HCC, while radiologists with various levels of specialisation made a certain number of errors. Radiologists misclassified HCC lesions with unclear washout as FNH or ICC lesions and HCC lesions with faint enhancement as metastases. The model could correctly identify HCC lesions by learning from the images. Saliency maps showed arterial enhancement on LAP, wash out on PVP and DP, and an enhancing capsule on DP, which was consistent with the major imaging features of LI-RADS. This result indicates that AI could aid radiologists and reduce the occurrence of misdiagnosis in clinical work. Meanwhile, saliency maps could help radiologists verify the predictions of the model and help clinicians understand the model performance.

The model showed poor performance in abscesses and metastases. A saliency map of abscesses showed that the model fixed its attention to the pattern of peripheral enhancement. However, there were many overlapping imaging features between abscesses and malignancies (Additional file 1: Fig. S2). In addition, with the progression of abscesses, there will be a variety of imaging features [25]. Primary malignancies with different biological behaviours and pathological changes along with tumour growth will affect the imaging features of metastases. Because the metastases in our study had different origins, the features were different [26]. The enhancement pattern of metastases is affected by nodule size, tumour vascularity and pathological behaviour changes with tumour growth. Small metastases may show hyperenhancement, while larger tumours may show intranodular necrosis or vascular thrombosis [27]. Hence, the model showed poor performance in small abscesses and metastases. In addition, a few typical lesions were misclassified by the model, which indicates that the performance of the model still has room for improvement.

Confusion matrices showed that the sources of diagnostic errors for the model and the academic radiologists for each disease were similar. To maintain consistency with the model, the radiologists diagnosed the lesions by reading the images, including the lesions and the surrounding part of the liver parenchyma, without reference to the related medical history or laboratory test results, which might affect the diagnostic accuracy. Radiologists could improve the accuracy by referring to clinical information. Therefore, we speculate that if clinical information and laboratory test results were included in the training process of the model, the diagnosis accuracy and the reliability of interpretation could also be effectively improved.

Our model is based on 3D-CNN. 2D-CNN is based on the assumption that the lesion grows and shrinks in a symmetrical and spherical manner, which is not accurate [28]. 3D CNN can accurately reflect the actual size of the tumour [29], evaluate the asymmetry of the tumour morphology [30] and learn the tissue characteristics of the lesion on MRI. In addition, our model was trained with seven-sequence images that were obtained from 5 types of MRI scanners and included more than 500 lesions. Fatty liver and cirrhosis did not affect the model performance. The model was reliable, robust and predictive. Although the total number of lesions was lower than that in a previous study [9], the model still showed similar performance. In addition, our network involved 

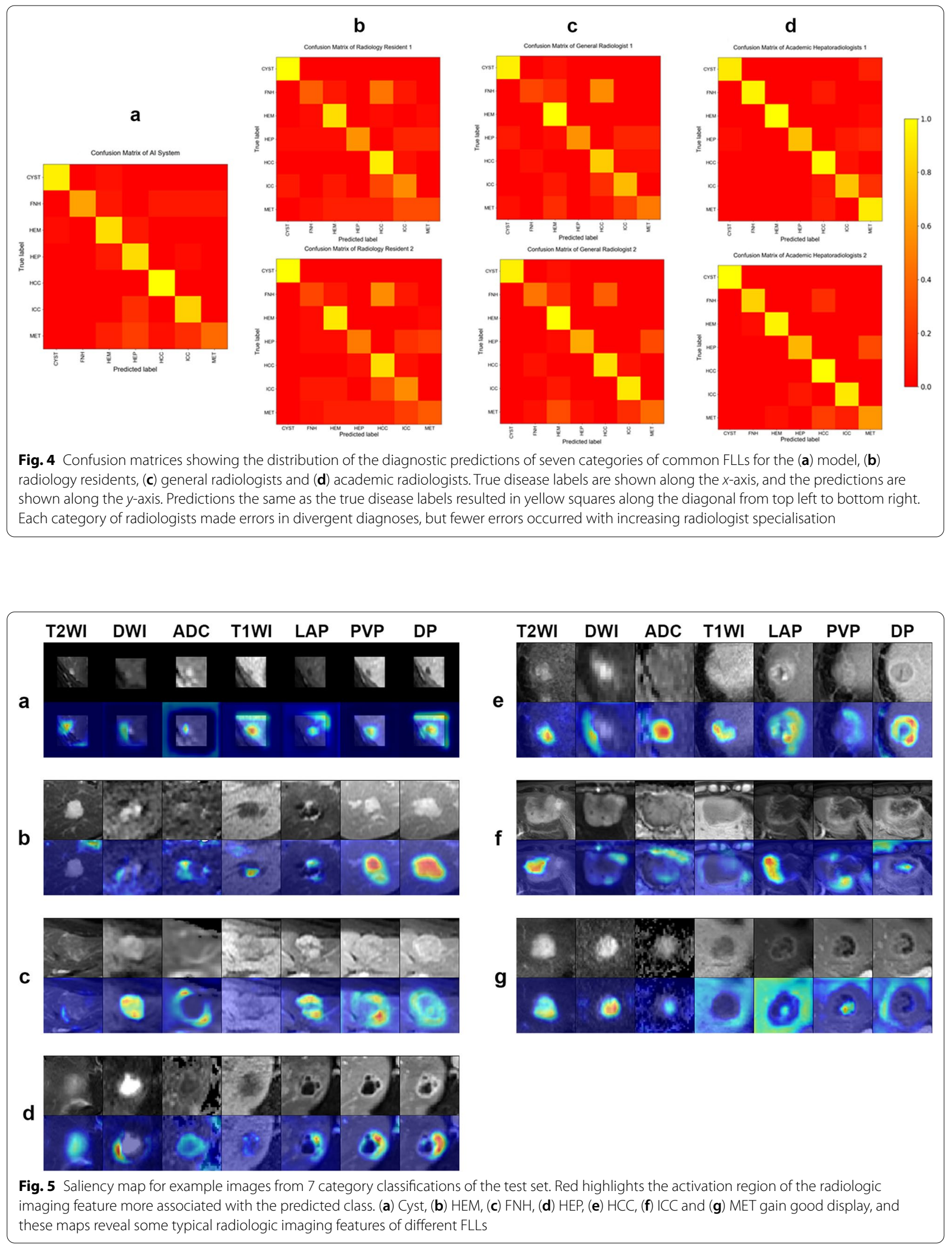


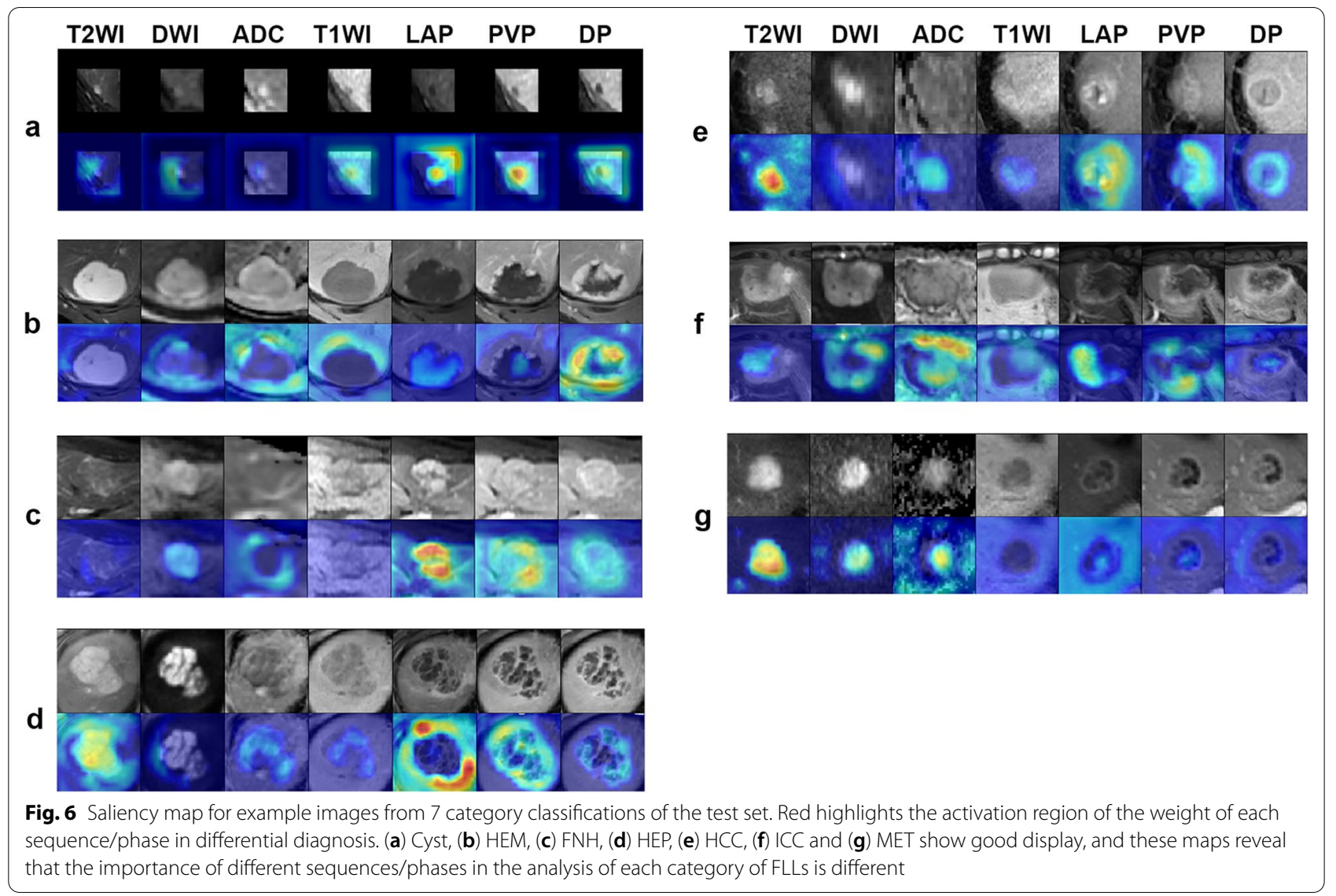

an attention mechanism, in which the boundary of the tumour was given more attention and then the network learned more representative features to achieve better diagnostic performance. A multitask framework was applied in our study to improve the learning efficiency, potential prediction accuracy and overfitting problems.

The previous study did not include the image information of unenhanced sequences such as T2WI, DWI and ADC. A comprehensive liver MRI protocol needs to evaluate the parenchyma, vasculature and biliary system, which is accomplished by way of a combination of unenhanced sequences and enhanced phases [31]. T2WI with fat suppression represents information about fluid content and fibrotic tissue and increases lesion conspicuity [32]. DWI and ADC can detect and characterise focal liver lesions and evaluate posttreatment changes in the tumour microenvironment [33]. T1WIs are acquired, which provide information regarding the $\mathrm{T} 1$ characteristics of lesions and serve as a baseline to evaluate enhanced phases [34]. The lack of unenhanced imaging could not fully evaluate the characteristics of FLL.

Although the classification models in the previous study had good performance, they were difficult to visualise and interpret. However, it is critical to explain model decision-making and let radiologists and clinicians verify the diagnosis [35]. Hence, we straightforwardly displayed interesting slices of 3D feature maps on the image containing the maximum area of the lesion and generated a radiologic imaging feature-based saliency map and sequence/phase weight-based saliency map. The radiologic imaging feature-based saliency map highlights the activation region of the radiologic imaging feature more associated with the predicted class. The sequence/phase weight-based saliency map highlights which image feature is more advantageous in classification by evaluating the importance of each sequence/phase. We showed that applying visualisation methods is important to understand the decisions of the model and is a step that is crucial to increase clinical impact and trust in deep learning models.

Our study has several limitations. First, our study focused on seven common categories of FLLs, while the types of FLLs in clinical practice are more extensive. Deep learning requires a large number of samples for model training. Due to the small number of some type of FLLs, it is difficult to train the model. Therefore, we only included 7 types of common FLLs for a preliminary model to reduce the daily work burden of radiologists. In the future, more patients 
with different types of FLLs (such as cirrhosis nodules and other rare liver tumours) need to be included to render the model applicable for different disease spectra in clinical practice. We will add validation datasets obtained from external centres to make the model more generalisable and reliable. Second, metastases from different primary origins in our study had different imaging features. Therefore, the model could not learn the lesion characteristics well, and its performance was poor. In the future, we need to increase the number of metastases in training or categorise them by their sources. Third, our study was a single-centre study, and we only used one type of intravenous contrast agent, which may limit the applicability of the model. In the future, we need to collect images from different hospitals using different contrast agents to make the model widely applicable. Fourth, saliency maps only evaluated the importance of a single sequence/phase in diagnosis but did not evaluate sequence/phase combinations. In the process of diagnosis, we need to consider the characteristics of lesions reflected by different sequences and phases on MRI. Therefore, we will continue to analyse the importance of sequence/phase combinations in the future.

\section{Conclusion}

This interpretable deep learning model showed high diagnostic performance in the differentiation of liver masses on multisequence MRI and used a saliency map to explain the analysis principle contributing to predictions, which made it more reliable. Due to the increasing demand for medical imaging in clinics and the different levels of radiologists in different regions, we expect that deep learning models could reduce the daily workload and may be in demand in radiology departments [36].

\begin{abstract}
Abbreviations
3D: Three-dimensional; ADC: Apparent diffusion coefficient; Al: Artificial intelligence; AUC: Area under the receiver operating characteristic curve; Cls: Confidence intervals; CNN: Convolutional neural network; CT: Computed tomography; DP: Delayed phase; DWI: Diffusion-weighted imaging; FC: Fully connected layers; FLL: Focal liver lesions; FNH: Focal nodular hyperplasia; HCC: Hepatocellular carcinoma; HEM: Cavernous haemangioma; HEP: Hepatic abscess; ICC: Intrahepatic cholangiocarcinoma; IQR: Interquartile range; LAP: Late arterial phase; LI-RADS: Liver imaging reporting and data system; MET: Hepatic metastasis; MRI: Magnetic resonance imaging; NLR: Negative diagnostic likelihood ratio; NPV: Negative predictive value; $A P^{*} A C S$ : Picture archiving and communication system; PLR: Positive diagnostic likelihood ratio; PPV: Positive predictive value; PVP: Portal venous phase; ROC: Receiver operating characteristic; T2Wl: T2-weighted imaging.
\end{abstract}

\section{Supplementary Information}

The online version contains supplementary material available at https://doi. org/10.1186/s13244-021-01117-z.

Additional file 1: Figure S1. Flowchart showing study selection according to the inclusion and exclusion criteria, from initial patient search to training-validation and test dataset randomisation. Figure S2 Axial MRI of a 55-year-old man with an abscess. (a) T2WI showed an irregularly shaped, hyperintense neoplasm in segment $\mathrm{V}$. The lesion showed (b) hyperintensity on DWI (c) with a low ADC, (d) hypointensity on T1WI, and (e) targetoid rim enhancement in the LAP, (f) PVP and (g) DP. The lesion was mistaken as ICC by the AI model.

\section{Authors' contributions}

SHW, XJH, JD, ZHY and HX contributed to study design, data acquisition, data analysis and manuscript preparation. ZCW and CWY contributed to data acquisition and manuscript final approval. YJZ, YNC, XDou and XWX contributed to technical development and data analysis. All authors read and approved the final manuscript.

\section{Funding}

This study was supported by the following grant: National Natural Science Foundation of China (No. 61871276,82071876); National Key R\&D Program of China (No. 2016YFC0106900); Beijing Natural Science Foundation (No. 7184199); Capital's Funds for Health Improvement and Research (No. 2018-2-2023); Capital Health Research and Development of Special Fund (No.2018-2-2182); Beijing Municipal Science \& Technology Commission (No. Z181100001718070); Beijing Hospitals Authority Clinical Medicine Development of Special Funding Support (No. ZYLX202101).

\section{Availability of data and materials}

The datasets used or analysed during the current study are available from the corresponding author on reasonable request.

\section{Declarations}

\section{Ethics approval and consent to participate}

This is not a research paper involving human participants and/or animals; informed consent is not required.

\section{Consent for publication}

Not applicable.

\section{Competing interests}

The authors confirm that there are no known conflicts of interest associated with this study.

\section{Author details}

${ }^{1}$ Department of Radiology, Beijing Friendship Hospital, Capital Medical University, No. 95 Yongan Road, Xicheng District, Beijing 100050, People's Republic of China. ${ }^{2}$ Department of Radiology, Weihai Municipal Hospital, Cheeloo College of Medicine, Shandong University, Weihai, Shandong Province, People's Republic of China. ${ }^{3}$ Center of Interventional Oncology and Liver Diseases, Beijing Youan Hospital, Capital Medical University, Beijing, People's Republic of China. ${ }^{4}$ SenseTime Research, SenseTime, Shanghai, People's Republic of China. ${ }^{5}$ WCH-SenseTime Joint Lab, SenseTime, Shanghai, Sichuan, People's Republic of China. 'SenseBrain Technology, SenseTime, Princeton, NJ 08540, USA.

Received: 19 September 2021 Accepted: 26 October 2021

Published online: 24 November 2021

\section{References}

1. Matos AP, Velloni F, Ramalho M, AlObaidy M, Rajapaksha A, Semelka RC (2015) Focal liver lesions: practical magnetic resonance imaging approach. World J Hepatol 7:1987-2008

2. van den Bos IC, Hussain SM, de Man RA et al (2008) Magnetic resonance imaging of liver lesions: exceptions and atypical lesions. Curr Probl Diagn Radiol 37:95-103

3. Strassburg CP, Manns MP (2006) Approaches to liver biopsy techniquesrevisited. Semin Liver Dis 26:318-327

4. Zhou LQ, Wang JY, Yu SY et al (2019) Artificial intelligence in medical imaging of the liver. World J Gastroenterol 25:672-682 
5. Jacobson FL (2020) Medical image perception research in the emerging age of artificial intelligence. Radiology 294:210-211

6. To MNN, Vu DQ, Turkbey B, Choyke PL, Kwak JT (2018) Deep dense multipath neural network for prostate segmentation in magnetic resonance imaging. Int J Comput Assist Radiol Surg 13:1687-1696

7. LeCun Y, Bengio Y, Hinton G (2015) Deep learning. Nature 521:436-444

8. Anwar SM, Majid M, Qayyum A, Awais M, Alnowami M, Khan MK (2018) Medical image analysis using convolutional neural networks: a review. J Med Syst 42:226

9. Zhen SH, Cheng M, Tao YB et al (2020) deep learning for accurate diagnosis of liver tumor based on magnetic resonance imaging and clinical data. Front Oncol 10:680

10. Yasaka K, Akai H, Abe O, Kiryu S (2018) Deep learning with convolutional neural network for differentiation of liver masses at dynamic contrastenhanced CT: a preliminary study. Radiology 286:887-896

11. Zhou J, Wang W, Lei B et al (2020) Automatic detection and classification of focal liver lesions based on deep convolutional neural networks: a preliminary study. Front Oncol 10:581210

12. Ortiz-Ramon R, Larroza A, Arana E, Moratal D (2017) A radiomics evaluation of $2 \mathrm{D}$ and $3 \mathrm{D}$ MRI texture features to classify brain metastases from lung cancer and melanoma. Annu Int Conf IEEE Eng Med Biol Soc 2017:493-496

13. Depeursinge A, Foncubierta-Rodriguez A, Van De Ville D, Muller H (2014) Three-dimensional solid texture analysis in biomedical imaging: review and opportunities. Med Image Anal 18:176-196

14. He K, Zhang X, Ren S, Sun J (2016) Deep residual learning for image recognition. In: 2016 IEEE conference on computer vision and pattern recognition (CVPR). IEEE, Las Vegas, pp 770-778

15. Erkan B, Meier J, Clark TJ, Kaplan J, Lambert JR, Chang S (2019) Noninvasive diagnostic criteria of hepatocellular carcinoma: comparison of diagnostic accuracy of updated LI-RADS with clinical practice guidelines of OPTN-UNOS, AASLD, NCCN, EASL-EORTC, and KLSCG-NCC. PLoS One 14:e226291

16. Jiang HY, Chen J, Xia CC, Cao LK, Duan T, Song B (2018) Noninvasive imaging of hepatocellular carcinoma: from diagnosis to prognosis. World J Gastroenterol 24:2348-2362

17. Mitchell DG, Bruix J, Sherman M, Sirlin CB (2015) LI-RADS (liver imaging reporting and data system): summary, discussion, and consensus of the LI-RADS management working group and future directions. Hepatology 61:1056-1065

18. Roberts LR, Sirlin CB, Zaiem F et al (2018) Imaging for the diagnosis of hepatocellular carcinoma: a systematic review and meta-analysis. Hepatology 67:401-421

19. Burns PN, Wilson SR (2007) Focal liver masses: enhancement patterns on contrast-enhanced images-concordance of US scans with CT scans and MR images. Radiology 242:162-174

20. Elsayes K, Hooker J, Agrons M et al (2017) 2017 version of LI-RADS for CT and MR imaging: an update. Radiographics 37:1994-2017

21. Barth BK, Donati OF, Fischer MA et al (2016) Reliability, validity, and reader acceptance of LI-RADS-an in-depth analysis. Acad Radiol 23:1145-1153
22. Ehman EC Behr SC Umetsu SE et al (2016) Rate of observation and inter-observer agreement for LI-RADS major features at CT and MRI in 184 pathology proven hepatocellular carcinomas. Abdom Radiol (NY) 41:963-969

23. Becker AS, Barth BK, Marquez PH et al (2017) Increased interreader agreement in diagnosis of hepatocellular carcinoma using an adapted LI-RADS algorithm. Eur J Radiol 86:33-40

24. Kierans AS, Makkar J, Guniganti P et al (2019) Validation of liver imaging reporting and data system 2017 (LI-RADS) criteria for imaging diagnosis of hepatocellular carcinoma. J Magn Reson Imaging 49:e205-e215

25. Park HJ, Kim SH, Jang KM, Lee SJ, Park MJ, Choi D (2013) Differentiating hepatic abscess from malignant mimickers: value of diffusion-weighted imaging with an emphasis on the periphery of the lesion. J Magn Reson Imaging 38:1333-1341

26. Dănilă M, Popescu A, Sirli R, Sporea I, Martie A, Sendroiu M (2010) Contrast enhanced ultrasound (CEUS) in the evaluation of liver metastases. Med Ultrason 12:233-237

27. Kong WT, Ji ZB, Wang WP, Cai H, Huang BJ, Ding H (2016) Evaluation of liver metastases using contrast-enhanced ultrasound: enhancement patterns and influencing factors. Gut Liver 10:283-287

28. Mantatzis M, Kakolyris S, Amarantidis K, Karayiannakis A, Prassopoulos P (2009) Treatment response classification of liver metastatic disease evaluated on imaging. Are RECIST unidimensional measurements accurate? Eur Radiol 19:1809-1816

29. Chapiro J, Lin M, Duran R, Schernthaner RE, Geschwind JF (2015) Assessing tumor response after loco-regional liver cancer therapies: the role of 3D MRI. Expert Rev Anticancer Ther 15:199-205

30. Chapiro J, Wood LD, Lin M et al (2014) Radiologic-pathologic analysis of contrast-enhanced and diffusion-weighted MR imaging in patients with HCC after TACE: diagnostic accuracy of 3D quantitative image analysis. Radiology 273:746-758

31. Fowler KJ, Brown JJ, Narra VR (2011) Magnetic resonance imaging of focal liver lesions: approach to imaging diagnosis. Hepatology 54:2227-2237

32. Donato H, Franca M, Candelaria I, Caseiro-Alves F (2017) Liver MRI: from basic protocol to advanced techniques. Eur J Radiol 93:30-39

33. Qayyum A (2009) Diffusion-weighted imaging in the abdomen and pelvis: concepts and applications. Radiographics 29:1797-1810

34. Lamba R, Fananapazir G, Corwin MT, Khatri VP (2014) Diagnostic imaging of hepatic lesions in adults. Surg Oncol Clin N Am 23:789-820

35. Bluemke DA, Moy L, Bredella MA et al (2020) Assessing radiology research on artificial intelligence: a brief guide for authors, reviewers, and readersfrom the radiology editorial board. Radiology 294:487-489

36. Wong SH, Al-Hasani H, Alam Z, Alam A (2019) Artificial intelligence in radiology: How will we be affected? Eur Radiol 29:141-143

\section{Publisher's Note}

Springer Nature remains neutral with regard to jurisdictional claims in published maps and institutional affiliations.

\section{Submit your manuscript to a SpringerOpen ${ }^{\circ}$ journal and benefit from:}

- Convenient online submission

- Rigorous peer review

- Open access: articles freely available online

- High visibility within the field

- Retaining the copyright to your article

Submit your next manuscript at $\boldsymbol{\nabla}$ springeropen.com 